MATHEMATICS OF COMPUTATION

Volume 70, Number 236, Pages 1549-1567

S $0025-5718(01) 01326-6$

Article electronically published on May 14, 2001

\title{
THREE- AND FOUR-DIMENSIONAL $K$-OPTIMAL LATTICE RULES OF MODERATE TRIGONOMETRIC DEGREE
}

\author{
RONALD COOLS AND JAMES N. LYNESS
}

\begin{abstract}
A systematic search for optimal lattice rules of specified trigonometric degree $d$ over the hypercube $[0,1)^{s}$ has been undertaken. The search is restricted to a population $K(s, \delta)$ of lattice rules $Q(\Lambda)$. This includes those where the dual lattice $\Lambda^{\perp}$ may be generated by $s$ points $\mathbf{h}$ for each of which $|\mathbf{h}|=\delta=d+1$. The underlying theory, which suggests that such a restriction might be helpful, is presented. The general character of the search is described, and, for $s=3, d \leq 29$ and $s=4, d \leq 23$, a list of $K$-optimal rules is given. It is not known whether these are also optimal rules in the general sense; this matter is discussed.
\end{abstract}

\section{INTRODUCTION}

We consider cubature rules for $[0,1)^{s}$ of trigonometric degree $d$. Such a rule integrates correctly all $s$-dimensional trigonometric polynomials of degree $d$. Specifically, it integrates $\exp (2 \pi i \mathbf{h} \cdot \mathbf{x})$ correctly for all $\mathbf{h}:=\left(h_{1}, h_{2}, \ldots, h_{s}\right) \in \mathbb{Z}^{s}$ that satisfy $|\mathbf{h}|:=\sum_{k=1}^{s}\left|h_{k}\right| \leq d$. Lattice rules have played a significant role in the development of this area. For background information of a general nature on lattice rules, we refer to SJ94 and to CS96 for lattice and other rules of specified trigonometric degree.

Definition 1.1. An $s$-dimensional lattice rule is a cubature formula that can be expressed in the form

$$
\begin{aligned}
Q f & =Q[t, D, Z, s] f \\
& :=\frac{1}{d_{1} d_{2} \ldots d_{t}} \sum_{j_{1}=1}^{d_{1}} \sum_{j_{2}=1}^{d_{2}} \ldots \sum_{j_{t}=1}^{d_{t}} f\left(\left\{\frac{j_{1} \mathbf{z}_{1}}{d_{1}}+\frac{j_{2} \mathbf{z}_{2}}{d_{2}}+\ldots+\frac{j_{t} \mathbf{z}_{t}}{d_{t}}\right\}\right),
\end{aligned}
$$

where $d_{i}$ are positive integers and $\mathbf{z}_{i} \in \mathbb{Z}^{s}$ for all $i$.

In this theory it is conventional to refer to $\mathbb{Z}^{s}$ (the set of points all of whose components are integers) as the $s$-dimensional unit lattice denoted by $\Lambda_{0}^{s}$. The abscissas of the lattice rule $Q f$ lie on an integration lattice $\Lambda$, that is, a discrete subset of $\mathbb{R}^{s}$ that is closed under addition and subtraction and that contains $\Lambda_{0}^{s}$. The arguments in the right-hand member in 1.1 may be assembled into two matrices.

Received by the editor November 29, 1999.

2000 Mathematics Subject Classification. Primary 41A55, 41A63, 42A10; Secondary 65D32.

The second author was supported by the Mathematical, Information, and Computational Sciences Division subprogram of the Office of Advanced Scientific Computing Research, U.S. Dept. of Energy, under Contract W-31-109-Eng-38.

(C)2001 University of Chicago \& Katholieke Universiteit Leuven 
These are the $t \times t$ matrix $D=\operatorname{diag}\left\{d_{i}\right\}$ and the $t \times s$ matrix $Z$ whose $i$ th row is $\mathbf{z}_{i}$. The rank and invariants of a lattice rule play no major role in the theory treated in this paper, and their definitions are omitted. However, we remark that much of the previous work in this area has been restricted to rank-1 simple lattice rules. These are rules that can be expressed in form (1.1) above with $t=1$ and $\mathbf{z}_{1}$ having 1 as its first component.

All cubature rules $Q$ have an abscissa count $N(Q)$ and have a trigonometric degree, say, $d(Q)$. It turns out to be more convenient to work with

$$
\delta:=d+1
$$

which we term the enhanced degree. An optimal rule of enhanced degree $\delta$ is one whose abscissa count is known to be as small as or smaller than the abscissa count $N\left(Q^{\prime}\right)$ of any other rule $Q^{\prime}$ of this same enhanced degree $\delta$. In this case we denote this count by $N_{\min }(s, \delta)$. A standard goal, which is our ultimate goal, is to find optimal rules.

Optimal rules are already known for $s=1$ and 2 for all $\delta$; they are also known for all $s$ with $\delta=1,2,3,4$ and for $(s, \delta)=(3,6)$. In each of these cases, at least one of these optimal rules is a lattice rule. Except in the cases just mentioned, no rule is known to be optimal, and it is not known whether there is any case in which one of the optimal rules is not a lattice rule.

A lower bound on $N_{\min }(s, \delta)$, which is based on the character of the set of moment equations, appears in CS96], is denoted here by $N_{M E}(s, \delta)$, and is available for all $s$ and $d$; however, except in the aforementioned cases, it is not known whether this bound is attained. In particular:

$$
\begin{array}{rlll}
N_{M E}(1, \delta) & =\delta & \\
N_{M E}(2, \delta) & =\delta^{2} / 2 & & \delta \text { even } \\
& =\left(\delta^{2}+1\right) / 2 & & \delta \text { odd } \\
N_{M E}(3, \delta) & =\delta\left(\delta^{2}+2\right) / 6 & & \delta \text { even } \\
& =\delta\left(\delta^{2}+5\right) / 6 & & \delta \text { odd } \\
N_{M E}(4, \delta) & =\delta^{2}\left(\delta^{2}+8\right) / 24 & & \delta \text { even } \\
& =\left(\delta^{4}+14 \delta^{2}+9\right) / 24 & & \delta \text { odd }
\end{array}
$$

A completely different bound, valid only for lattice rules, follows from applying Minkowski's celebrated theorem about admissible lattices to an $s$-dimensional octahedron $\Omega(s, \delta)$ defined in (2.3). In the present context, this provides a bound $N \geq \delta^{s} / s$ ! for lattice rules. A much deeper result of his introduces a "critical lattice" for $s=1,2$, and 3 . The consequence for us is that a bound exists that is specific for lattice rules:

$$
N \geq \bar{N}_{C L}(s, \delta):=\frac{\delta^{s}}{s ! \theta(s)} .
$$

Clearly $\theta(s) \leq 1$. The only known values of $\theta(s)$ are $\theta(1)=\theta(2)=1$ and $\theta(3)=$ 18/19. In the literature on geometry of numbers [GL87], $\theta(s)$ is known as the "density of closest (or densest) lattice packing" for the $s$-dimensional octahedron. Nontrivial upper bounds for $\theta(s), s \geq 4$, appear to be unknown. Every lattice rule provides a lower bound for $\theta(s)$. Examination of our recent results in Table 2 establishes $\theta(4) \geq \frac{512}{621}$. This improves the result of Klyuchnikov and Reztsov KR95, $\theta(4) \geq \frac{128}{159}$, by a margin of approximately 0.02 . In our context, $N$ is an 
integer, so the above inequality may be sharpened to

$$
N \geq N_{C L}(s, \delta):=\left\lceil\bar{N}_{C L}(s, \delta)\right\rceil .
$$

For $s=1$ and $2, N_{C L}=N_{M E}$. For $s=3$ and $\delta=8$ and $\geq 10, N_{C L} \geq N_{M E}$. Numerical values of $N_{M E}$ and $N_{C L}$ are given in Tables 1 and 2 ,

A small amount of literature (mostly in Russian) has been devoted to optimal cubature rules. The optimal rules mentioned above appear in papers by Mysovskikh Mys85, Mys87, Mys88 and Noskov [Nos85, Nos88a, [Nos88b] and are elaborated by Beckers and Cools [BC93], Cools and Sloan [CS96] and Cools and Reztsov CR97. The three-dimensional rule is classical and due to Minkowski (see [Fro77] and [Min67, Chapter XIX].

Furthermore, Noskov and Semenova have published many nonoptimal individual three-, four-, and five-dimensional rank-1 simple lattice rules and several families; see, for example, Nos88a, Nos91, NS96, Sem96]. Each family is a one-parameter system (the parameter being essentially the degree) of rank-1 simple lattice rules; and, since the parameter is unbounded, these include rules of arbitrarily high degree. It is not revealed how they were discovered, but clearly careful effort was expended, and they are far more economical than those (such as the center and vertex rule) previously available. However, it appears that none is likely to be particularly close to optimal. To our knowledge these are the only lattice rules available that are reasonably efficient from the trigonometric point of view.

We have carried out a large-scale computer search with a view to clarifying the situation as far as optimal lattice rules in dimensions 3 and 4 are concerned. We have managed to reach degree 30 in three dimensions and to reach degree 24 in four. In this paper, we describe this search and give some background in the context of other analogous searches. We present some of the results.

Our search is however restricted to a subset of the lattice rules, namely, $K(s, \delta)$ of Definition 2.7 below. There are compelling reasons for believing that the optimal lattice rules are members of this set, but this has not been proved. We have come across no counterexample nor any suggestion that such a counterexample may exist. Nevertheless, we retain the distinction and refer to the optimal lattice rules of this set as $K$-optimal lattice rules.

\section{UNDERLYING THEORY}

The theory on which our search is based is closely analogous to the theory on which some searches for good lattices are based. We give a brief description here, mainly to introduce the standard notation.

A lattice $\Lambda$ may be defined in terms of an $s \times s$ matrix $A$ known as a generator matrix. This means that all elements of $\Lambda$ are of the form $x=\lambda A$, where $\lambda \in \mathbb{Z}^{s}$. The dual lattice $\Lambda^{\perp}$ may be defined as one having generator matrix $B=\left(A^{T}\right)^{-1}$. The reader will recall that, since $\Lambda$ is an integration lattice, that is, $\Lambda \supseteq \Lambda_{0}^{s}$, its dual $\Lambda^{\perp}$ is an integer lattice and may be generated by an integer-valued matrix $B$.

When $U$ is any unimodular matrix, $H=U B$ is also a generator matrix for $\Lambda^{\perp}$. For any given $B$, there exists a particular choice for $U$ that will provide a generator matrix $H=U B$ that is in Hermite normal form (utlf). That is,

$$
\begin{array}{ll}
H_{c, c}>0 & \\
H_{r, c}=0 & r>c \\
H_{r, c} \in\left[0, H_{c, c}\right) & r<c .
\end{array}
$$


A (1-1) correspondence exists between the set of $s$-dimensional lattice rules and the set of $s \times s$ matrices in Hermite normal form. This has been exploited previously to organize searches [LS93] but is not exploited in that way here.

The quantity $|\operatorname{det} B|$ is conventionally known as the order of the lattice $\Lambda^{\perp}$. The simplex whose $s+1$ vertices make up the $s$ rows of $B$, namely, $\mathbf{b}_{j}(j=1,2, \ldots, s)$, together with the origin $\mathbf{O}$ is known as a basic cell of $\Lambda^{\perp}$. (Any simplex obtained in this way using any generating matrix $U B$ is also a basic cell, as is any simplex obtained by translating one of these simplices.) The $s$-volume of a basic cell is $|\operatorname{det} B| / s$ !. In fact, all $s$-dimensional simplectical regions whose vertices are distinct elements of $\Lambda^{\perp}$ have $s$-volume $k|\operatorname{det} B| / s$ !, where $k$ is some nonnegative integer. (Any set of vertices for which $k=1$ forms a basic cell.) The relevance of the basic cell to our search lies in the fact that the abscissa count of $Q$ coincides with $|\operatorname{det} B|$ (see [Lyn89]), that is,

$$
N(Q(\Lambda))=|\operatorname{det} B|=\prod_{i=1}^{s} H_{i, i} .
$$

This may be reexpressed as follows.

Theorem 2.1. The abscissa count $N$ of $Q(\Lambda)$ coincides with the order of $\Lambda^{\perp}$.

When $Q(\Lambda)$ is the lattice rule whose integration lattice is $\Lambda$, the associated Poisson summation formula reduces to an expression for the discretization error, namely,

$$
E_{Q(\Lambda)} f:=Q(\Lambda) f-I f=\sum_{\substack{\mathbf{h} \in \Lambda^{\perp} \\ \mathbf{h} \neq \mathbf{0}}} \hat{f}_{\mathbf{h}},
$$

where $\hat{f}_{\mathbf{h}}$ is the Fourier coefficient of $f$ and $\Lambda^{\perp}$ is the dual lattice of $\Lambda$. When $f$ is a trigonometric polynomial of degree $d$ or less, $\hat{f}_{\mathbf{h}}=0$ when $|\mathbf{h}|>d$, so all but a finite set of terms in this sum vanish. Thus, the condition that $Q(\Lambda) f$ is exact for these polynomials reduces to the condition that $\Lambda^{\perp}$ has no elements, other than the origin itself, in the region $|\mathbf{h}| \leq d$, which we denote by $\Omega(s, d)$. We may restate this as follows:

$$
\delta(Q(\Lambda)):=d(Q(\Lambda))+1=\min _{\substack{\mathbf{h} \in \Lambda^{\perp} \\ \mathbf{h} \neq \mathbf{0}}}|\mathbf{h}| .
$$

This equation relates the location of points $\mathbf{h} \in \Lambda^{\perp}$ with the enhanced degree $\delta$ of $Q(\Lambda)$. We may use classical terminology to reexpress the import of this equation in terms taken from the geometry of numbers GL87.

Definition 2.2. (Classical) A lattice $L$ is "admissible" with respect to a region $\Omega$ if all its elements (other than the origin) lie outside $\Omega$.

Such a lattice is conventionally known as an $\Omega$-admissible lattice. Applied to our region

$$
\mathbf{h} \in \Omega(s, \delta) \text { when }|\mathbf{h}| \leq \delta,
$$

we have the following definition.

Definition 2.3. An $\Omega(s, \delta)$-admissible lattice is an integer lattice having no elements, other than the origin, in the interior of $\Omega(s, \delta)$.

Using this terminology, we may write the content of (2.2) as follows: 
Theorem 2.4. $Q(\Lambda)$ is of enhanced degree $\delta$ or greater if and only if $\Lambda^{\perp}$ is $\Omega(s, \delta)$ admissible.

This theorem, together with Theorem 2.1, leads to the following geometric characterization.

Theorem 2.5. $Q(\Lambda)$ is an optimal lattice rule of enhanced degree $\delta$ when $\Lambda^{\perp}$ is an $\Omega(s, \delta)$-admissible lattice and no other $\Omega(s, \delta)$-admissible lattice has a lower order.

In passing, it is pertinent to mention that many other criteria are in use to characterise efficient cubature rules, and that some, like the enhanced degree in (1.2) above, are based on exact evaluation of specified sets of Fourier coefficients. Some of these latter are discussed in Lyness Lyn88 and may be described in terms of $\Omega$-admissible lattices with $\Omega$ redefined appropriately. The two most familiar choices are illustrated in, e.g., BC93. Other choices are investigated in CR97. and [LS97.

We now return to the problem at hand. In this paper, $\Omega$ is defined in (2.3) and we are treating the enhanced degree, defined in (1.2).

A dynamic approach to the problem of finding an optimal rule might involve perturbing any given $\Omega(s, \delta)$-admissible lattice $\Lambda^{\perp}$, with a view to reducing the $s$-volume of its unit cell but keeping it $\Omega(s, \delta)$-admissible, that is, not allowing any lattice point to enter the fixed region $\Omega(s, \delta)$.

It is reasonable to believe that the process of making this unit cell small, that is, making the lattice $\Lambda^{\perp}$ denser and reducing its order, would, in general, move lattice points towards the origin. This process would be seriously inhibited by the boundary of $\Omega(s, \delta)$. Ultimately, (as the wiggle room disappears) one would expect progress to come to a complete stop (grind to a halt) at a stage where many points of $\Lambda^{\perp}$ were (jammed) on this boundary. Thus, it is plausible to believe that the lattice $\Lambda$ of an optimal lattice rule $Q(\Lambda)$ of enhanced degree $\delta$ will have a dual lattice $\Lambda^{\perp}$ with many elements on this boundary. The underlying feature of our search is that it is limited to dual lattices having this property.

The $(s-1)$-dimensional facet-pair of an $s$-crosspolytope is the $s$-dimensional generalization of a two-dimensional pair of opposite faces of a regular (threedimensional) octahedron. We recall the following notation:

$$
\begin{gathered}
|\mathbf{x}|=\left|\left(x_{1}, x_{2}, x_{3}, \ldots, x_{s}\right)\right|=\left|x_{1}\right|+\left|x_{2}\right|+\left|x_{3}\right|+\ldots+\left|x_{s}\right| \\
\mathbf{h} \in \Omega(s, \delta) \text { when }|\mathbf{h}| \leq \delta \\
\mathbf{h} \in \bar{\Omega}(s, \delta) \text { when }|\mathbf{h}|=\delta .
\end{gathered}
$$

In the sequel, $\sigma_{i}$ stands for +1 or for -1 .

Definition 2.6. The facet-pair $F\left(\delta, \sigma_{1}, \sigma_{2}, \sigma_{3}, \ldots, \sigma_{s}\right)$ comprises $\mathbf{h}$ satisfying

$$
\mathbf{h} \in \bar{\Omega}(s, \delta) \text { and }
$$

either

$$
h_{i}=\sigma_{i}\left|h_{i}\right| \text { for all } i=1,2,3, \ldots, s
$$

or

$$
h_{i}=-\sigma_{i}\left|h_{i}\right| \text { for all } i=1,2,3, \ldots, s .
$$


Definition 2.7. The population $K(s, \delta)$ comprises all $s$-dimensional lattices that may be generated by $s$ point pairs, each of which belongs to a distinct $(s-1)$ dimensional facet-pair of the $s$-octahedron (s-crosspolytope) $\Omega(s, \delta)$.

Note that a lattice in $K(s, \delta)$ cannot have enhanced degree exceeding $\delta$ because, by definition, it includes points $\mathbf{h}$ having $|\mathbf{h}|=\delta$. In general such a lattice is of degree less than $\delta$.

We search this population for the rule or rules defined as follows.

Definition 2.8. A $K(s, \delta)$-optimal rule is a rule of minimum abscissa count among those of enhanced degree $\delta$ whose dual lattice $\Lambda^{\perp}$ is in $K(s, \delta)$.

In the next section, we shall require subsets of $K(s, \delta)$. These will be denoted by $K(s, \delta ; X)$, where $\mathrm{X}$ will identify the particular subset in question.

\section{THE SEARCH PROGRAMS}

In this section we describe the implementation of programs based on the ideas and definitions introduced at the end of the preceding section. It has turned out that the four-dimensional program is significantly more complicated than the three-dimensional program. For this reason, after introducing some common $s$ dimensional notation, we describe the three-dimensional program first. Then, with the underlying ideas exposed in the simpler context, we treat the four-dimensional program.

In three or more dimensions, significant effort can be saved by exploiting the existence of sets of symmetrically equivalent lattices. A group of linear transformations takes the $s$-cube, or the $s$-octahedron, into itself. Applying one of these transformations to a rule or a lattice provides another (generally different) rule or lattice having the same geometric characteristics. Naturally, two lattices related in this way have the same (enhanced) degree and the same order (abscissa count). A set of symmetrically equivalent lattices may have as many as $s ! 2^{s-1}$ members. Once one member of such a set is established to be optimal, the other members of the set may be rapidly identified and are also optimal. Thus, if we are able to subdivide the search population in such a way that a search over one part will recover only symmetric equivalents of a search over another part, we may exploit this by searching only one of these parts. A search over the second part can be safely omitted, as it would reveal only optimal lattices that are symmetric equivalents of optimal lattices already identified.

In three dimensions, it is particularly easy to exploit the concept of sets of symmetrically equivalent lattices. In view of Definition 2.7 above, the set $K(3, \delta)$ includes all lattices generated by three points $\mathbf{b}_{1}, \mathbf{b}_{2}$, and $\mathbf{b}_{3}$, where each lies on a different facet-pair. We define a subset of $K(3, \delta)$, which we denote by $K^{*}$. This includes only lattices generated by

$$
\begin{aligned}
& \mathbf{b}_{1} \in F(\delta,+,+,+) \\
& \mathbf{b}_{2} \in F(\delta,+,+,-) \\
& \mathbf{b}_{3} \in F(\delta,+,-,+) .
\end{aligned}
$$

It is straightforward to show that all lattices in $K(3, \delta)$ have a symmetrically equivalent lattice in $K^{*}$. Thus, we may restrict our search to the elements of $K^{*}$ and then include, in addition, all symmetric equivalents. The outcome is the same as 
if we had treated all the elements of $K(3, \delta)$, but is obtained at approximately one fourth the cost. (The corresponding statement in four dimensions is not true.)

Our search module has two principal modes of operation. In mode 1 (its usual mode) it requires as input numerical values of $\delta$ and $N_{L}$ and $N_{U}$. It also requires a specification of the population to be treated. (When $s=3$, this is simply the set $K^{*}$ discussed above. For $s=4$, as described later, several different population specifications may be used in different runs.) It carries out a search over this population set and either

(A) provides the generator matrix of a lattice $\Lambda^{\perp}$ for which the rule $Q(\Lambda)$ is of enhanced degree $\delta$, the order $N$ of this rule satisfies $N \in\left[N_{L}, N_{U}\right]$, and there is no rule of lower order in this interval; or

(B) reports that no lattice $\Lambda^{\perp}$ of enhanced degree $\delta$ with $N \in\left[N_{L}, N_{U}\right]$ exists in the specified input population.

To obtain this information, the search module proceeds as follows. It carries out a loop over all matrices $B$ whose rows $\mathbf{b}_{1}, \mathbf{b}_{2}, \ldots, \mathbf{b}_{s}$ are elements of their respective facet-pairs (see (3.1) above). Thus, there are possibly $\left(\begin{array}{c}s+\delta-1 \\ s-1\end{array}\right)^{s}$ matrices $B$ to consider. For each, the order $|\operatorname{det} B|$ is evaluated. Unless $|\operatorname{det} B| \in\left[N_{L}, N_{U}\right]$, this matrix $B$ is abandoned, and the next matrix $B$ is treated.

In the relatively few cases in which $|\operatorname{det} B|$ is within these limits, an algorithm for determining the enhanced degree of $\Lambda^{\perp}$ (or an upper bound on this) is invoked. Unless this enhanced degree is $\delta$, this matrix $B$ is abandoned and the next one is treated. Should this enhanced degree turn out to be $\delta$, ipso facto one lattice satisfying (A) above is available. In mode 1 , the search immediately downgrades $N_{U}$ to $N-1$ and continues (unless $N=N_{L}$, in which case it stops).

In all cases, if the module encounters no $\Omega(s, \delta)$-admissible lattice of enhanced degree $\delta$, the conclusion (B) above is reported.

The module can also be run in mode 2 . This requires the same input as in mode 1. However, instead of downgrading $N_{U}$ to $N-1$ when one lattice satisfying (A) is encountered, it downgrades $N_{U}$ to $N$ and continues until all matrices $B$ have been treated. This mode is normally used when the optimal $N_{\text {opt }}$ has already been determined and is invoked to see whether there are several different solutions. One sets $N_{L}=N_{U}=N_{\text {opt }}$.

The list of rules in Table 3 was obtained as follows. For each value of $\delta$, the search module was used with $N_{U}$ large and $N_{L}=\max \left(N_{M E}(3, \delta), N_{C L}(3, \delta)\right)$ as given in (1.3) and (1.5). The value of $N$ returned in item (A) was used in a second run using mode 2. Finally, the list of matrices was processed to remove all symmetric equivalents. Note that, without the second run, one of the entries for each of $\delta=$ 5 and 11 in Table 3 would have been missed.

The 4-octahedron has eight facet-pairs.

$$
\begin{array}{lll}
F_{0} & F(\delta,+,+,+,+) & \mathrm{E} \\
F_{1} & F(\delta,-,+,+,+) & \mathrm{O} \\
F_{2} & F(\delta,+,-,+,+) & \mathrm{O} \\
F_{3} & F(\delta,-,-,+,+) & \mathrm{E} \\
F_{4} & F(\delta,+,+,-,+) & \mathrm{O} \\
F_{5} & F(\delta,-,+,-,+) & \mathrm{E} \\
F_{6} & F(\delta,+,-,-,+) & \mathrm{E} \\
F_{7} & F(\delta,-,-,-,+) & \mathrm{O}
\end{array}
$$


Each has been assigned a serial number, which appears as a subscript in column 1. For later convenience, in column 3 we have assigned a parity to each. $F\left(\delta, \sigma_{1}, \sigma_{2}, \sigma_{3}, \sigma_{4}\right)$ is of even parity $\mathrm{E}$ if the set $\left(\sigma_{1}, \sigma_{2}, \sigma_{3}, \sigma_{4}\right)$ contains an even number of elements +1 .

Every lattice $\Lambda^{\perp}$ in $K(4, \delta)$ is generated by four points on four distinct facetpairs. We term such a set of facet-pairs a quartet (of facet-pairs). If we were to take no account of the symmetric equivalents, we would need to treat every distinct quartet separately. There are seventy distinct quartets, this being the number of ways of choosing four facet-pairs from the total of eight facet-pairs listed above. The following discussion is devoted solely to establishing Theorem 3.4 below, which assures us that only four of these quartets need be searched to ensure that we recover at least one symmetric equivalent of every optimal rule.

Definition 3.1. An individual quartet, denoted by $q\left(N_{1}, N_{2}, N_{3}, N_{4}\right)$ where $0 \leq$ $N_{1}<N_{2}<N_{3}<N_{4} \leq 7$ comprises a set of four distinct facet-pairs $F_{N_{1}}, F_{N_{2}}, F_{N_{3}}$ and $F_{N_{4}}$.

The type of a quartet $q$ is $\min (N E, N O)$ where $N E$ is the number of even facetpairs and $N O$ is the number of odd facet-pairs in $q$. For example, $q(0,4,5,6)$ contains three even facet-pairs, namely, $F_{0}, F_{5}$, and $F_{6}$, together with one odd facet-pair, $F_{4}$. Thus its type is 1 , this being the minimum of $N E=3$ and $N O=1$.

Definition 3.2. Let $q\left(N_{1}, N_{2}, N_{3}, N_{4}\right)$ be one of these 70 quartets. The population $K(4, \delta ; q)$ comprises any lattice that may be generated by four points $\mathbf{b}_{i}$, where $\mathbf{b}_{i} \in F_{N_{i}}, i=1,2,3,4$.

The union of all seventy of these populations $K(4, \delta ; q)$ includes all lattices that may be generated by four distinct points, each of which lies on a distinct facet-pair of the 4-octahedron, and so coincides with $K(4, \delta)$.

Let $\mathcal{G}_{i}$ be an element of the group $\mathcal{G}$ of 384 affine transformations that take the 4-octahedron into itself. Specifically, this transformation takes any facet-pair $F_{N_{j}}$ into some other facet-pair $F_{N_{k}}$, which we may denote by $\mathcal{G}_{i} F_{N_{j}}$. By the same token, this transformation takes separately each of a set of four facet-pairs into another set of four facet-pairs.

Definition 3.3. Let $q=q\left(N_{1}, N_{2}, N_{3}, N_{4}\right)$ be one of these 70 quartets. The quartet comprising the four facet-pairs $\mathcal{G}_{i} F_{N_{j}}, j=1,2,3,4$, is termed a symmetric copy of $q\left(N_{1}, N_{2}, N_{3}, N_{4}\right)$ and is denoted by $\mathcal{G}_{i} q$.

Let $q_{1}$ stand for the quartet $q(0,2,4,7)$. Clearly, a search over $K\left(4, \delta ; \mathcal{G}_{i} q_{1}\right)$ will yield only lattices that are symmetrically equivalent to those obtained in the same search over $K\left(4, \delta ; q_{1}\right)$. It is a trivial calculation to obtain all symmetric equivalents of a particular lattice. Thus, carrying out a search over more than one quartet belonging to the set of quartets $\mathcal{G}_{i} q_{1}$ is unnecessary. A straightforward calculation (elaborated in the Appendix) reveals that there are only 32 distinct quartets of this form. Thus, $S_{1}$ is a set of order 32 , and we need to search over only one of these 32 quartets. Our choice for $q_{1}$ could be replaced by any other member of $S_{1}$ with the same result.

We repeat this operation starting with the three specific quartets given in the theorem.

Theorem 3.4. Let $q_{0}=q(1,2,4,7), q_{1}=q(0,2,4,7), q_{2 a}=q(0,2,4,6)$, and $q_{2 b}=$ $q(0,2,4,5)$ and the sets of quartets constituting symmetric copies of $q_{j}$ be denoted 
by $S_{j}$. Then the sets $S_{i}$ are mutually disjoint. They are of orders 2,32,12, and 24, respectively, and their union includes all seventy quartets.

Proof. The sets $S_{0}, S_{1}, S_{2 a}, S_{2 b}$, are listed in the Appendix. The theorem may be verified by four sets of 384 simple calculations. One calculates $q=\mathcal{G}_{i} q_{j}(i=$ $1, \ldots, 384), j=0,1,2 a, 2 b$, and verifies that each is a member of the expected set $S_{j}$. The reader will note that all elements of $S_{i}, i=0,1$, are of type $i$ and elements of $S_{2 a}$ and $S_{2 b}$ are of type 2. It is straightforward to show that none of the 384 transformations alters the type of the quartet. The Appendix provides further details.

The results of our computer searches for four-dimensional optimal rules are presented in Table 5. This is in three parts. For each value of $\delta \in[1,13]$ we have made four distinct runs and (unless there are calculational errors) we have a complete list of all optimal $K(4, \delta)$ rules.

For $\delta \in[14,17]$ we reduced the population to $K\left(4, \delta ; q_{1}\right)$, where, as before, $q_{1}=$ $q(0,2,4,7)$. This restriction to a single quartet reduces the overall run time by a factor of 4 .

Beyond $\delta=18$, even this became too time consuming, and we reduced the population once more to $K\left(4, \delta ; q_{1}^{+}\right)$. The symbol $q_{1}^{+}$is used here to denote a subset of $q_{1}$ that includes all of $F_{2}, F_{4}$, and $F_{7}$, but only the part of $F_{0}=F(\delta,+,+,+,+)$ for which $x_{1} \geq x_{2} \geq x_{3} \geq x_{4}$. This reduces the size of the population by a factor of up to 24 . But almost certainly some optimal rules are missed.

We have described the three searches above in terms of the results. In the order of implementation, we first carried out a search using population $K\left(4, \delta ; q_{1}^{+}\right)$for $\delta$ up to 24 . Next, we used $K\left(4, \delta ; q_{1}\right)$ for $\delta$ up to 17 . Finally, we carried out a complete search, using four choices for $q$, for $\delta$ up to 13 .

\section{NEW RESUlts}

In subsections 4.2 and 4.3 we present some of our three- and four-dimensional results, respectively. Subsection 4.1 is devoted to careful definitions of the notation used in the tables.

4.1. Abscissa counts. In this first subsection we present the progress toward determining $N_{\text {opt }}(s, \delta)$, the optimal abscissa count for any $s$-dimensional rule of enhanced trigonometric degree $\delta$. We have in general obtained well-defined bounds on this quantity. These are denoted by $N_{X}(s, \delta)$, where the subscript $X$ indicates a limitation to the class of rules considered.

The five principal abscissa count functions we have listed are as follows:

- $N_{M E}$ : A theoretical lower bound for any rule of enhanced degree $\delta$, based on the relevant Moment Equations.

- $N_{C L}$ : The Minkowski lower bound for any lattice rule of enhanced degree $\delta$, based on the existence of the critical lattice (known only for dimensions $s=1,2$ and 3$)$.

- $N_{K O}$ : The lowest count for any $K(s, \delta)$-optimal rule. (We also list variants of $\left.N_{K O}.\right)$

- $N_{r 1 s}$ : The lowest abscissa count for any optimal rank-1 simple rule.

- $N_{\text {prev }}$ : The lowest abscissa count for any rule published in references Nos88a, NS96. These are all rank-1 simple. 
TABLE 1. Three-dimensional abscissa counts

\begin{tabular}{|c|c|c|c|c|c|c|}
\hline $\begin{array}{r}\delta \\
=d+1 \\
\end{array}$ & $N_{M E}$ & $N_{C L}$ & $N_{K O}$ & $N_{r 1 s}$ & $N_{\text {prev }}$ & $\rho\left(N_{K O}\right)$ \\
\hline 1 & 1 & 1 & 1 & 1 & 1 & 0.167 \\
\hline 2 & 2 & 2 & 2 & 2 & 2 & 0.667 \\
\hline 3 & 7 & 5 & 7 & 7 & 7 & 0.643 \\
\hline 4 & 12 & 12 & 12 & 12 & 12 & 0.889 \\
\hline 5 & 25 & 22 & 27 & 27 & 27 & 0.772 \\
\hline 6 & 38 & 38 & 38 & 38 & 38 & $\underline{0.947}$ \\
\hline 7 & 63 & 61 & 70 & 70 & 70 & 0.817 \\
\hline 8 & 88 & 91 & 92 & 92 & 92 & 0.928 \\
\hline 9 & 129 & 129 & 144 & 145 & 145 & 0.844 \\
\hline 10 & 170 & 176 & 178 & 178 & 178 & 0.936 \\
\hline 11 & 231 & 235 & 260 & 260 & 260 & 0.853 \\
\hline 12 & 292 & 304 & 304 & 312 & 312 & $\underline{0.947}$ \\
\hline 13 & 377 & 387 & 421 & 421 & 421 & 0.870 \\
\hline 14 & 462 & 483 & 486 & 486 & 486 & 0.941 \\
\hline 15 & 575 & 594 & 635 & 635 & 635 & 0.886 \\
\hline 16 & 688 & 721 & 724 & 724 & 724 & 0.943 \\
\hline 17 & 833 & 865 & 921 & 921 & 921 & 0.889 \\
\hline 18 & 978 & 1026 & 1026 & 1038 & 1038 & $\underline{0.947}$ \\
\hline 19 & 1159 & 1207 & 1276 & 1276 & 1319 & 0.896 \\
\hline 20 & 1340 & 1408 & 1412 & 1412 & 1412 & 0.944 \\
\hline 21 & 1561 & 1630 & 1708 & 1723 & 1771 & 0.904 \\
\hline 22 & 1782 & 1874 & 1878 & 1878 & 1942 & 0.945 \\
\hline 23 & 2047 & 2141 & 2240 & 2255 & 2327 & 0.905 \\
\hline 24 & 2312 & 2432 & 2432 & 2448 & 2532 & $\underline{0.947}$ \\
\hline 25 & 2625 & 2749 & 2865 & 2865 & 2977 & 0.909 \\
\hline 26 & 2938 & 3093 & 3098 & 3098 & 3218 & 0.946 \\
\hline 27 & 3303 & 3463 & 3591 & 3591 & 3751 & 0.914 \\
\hline 28 & 3668 & 3862 & 3868 & 3868 & 4032 & 0.946 \\
\hline 29 & 4089 & 4291 & 4445 & 4445 & 4635 & 0.915 \\
\hline 30 & 4510 & 4750 & 4750 & 4770 & 4958 & 0.947 \\
\hline
\end{tabular}

Formulas for $N_{M E}$ are given for all $(s, \delta)$ in reference CS96 and repeated by us for $s \leq 4$ in (1.3) above. $N_{C L}$ is simply (1.5) above. The principal contribution of our work is the list of values of $N_{K O}$ and some variants in Tables 1 and 2 We obtained the fourth abscissa count $N_{r 1 s}$ for $s=3(\delta \leq 30)$ and $s=4(\delta \leq 13)$ using a simple search program not discussed here. The fifth abscissa count $N_{\text {prev }}$ is readily gleaned from the cited literature.

The three-dimensional abscissa counts listed in Table 1 are all precisely as defined above. The four-dimensional abscissa counts listed in Table 2 are also precisely as defined above for $\delta \leq 13$. For higher values of $\delta$, the entries under $N_{K O}$ refer to the results of restricted searches, as indicated in Table 5 and specified at the end of the preceding section. The corresponding entries under $N_{r 1 s}$ may not be optimal. Rules corresponding to every abscissa count given in the columns labeled $N_{K O}$ and $N_{r 1 s}$ are specified in Tables 3, 4, 5, and 6.

For odd $\delta>14$ some rules have been published, but these use more points than published rules of higher degree. We have omitted these. 
TABLE 2. Four-dimensional abscissa counts

\begin{tabular}{|r|r||r||r|r|r|}
\hline$\delta$ & $N_{M E}$ & $N_{K O}$ & $N_{r 1 s}$ & $N_{\text {prev }}$ & $\rho\left(N_{K O}\right)$ \\
\hline \hline 1 & 1 & & 1 & 1 & 0.042 \\
2 & 2 & & 2 & 2 & 0.333 \\
3 & 9 & & 9 & 9 & 0.375 \\
4 & 16 & & 16 & 16 & 0.667 \\
5 & 41 & 45 & 46 & 46 & 0.579 \\
6 & 66 & 68 & 70 & 70 & 0.794 \\
7 & 129 & 152 & 152 & 156 & 0.658 \\
8 & 192 & 212 & 212 & 212 & 0.805 \\
9 & 321 & 375 & 398 & 414 & 0.729 \\
10 & 450 & 516 & 522 & 522 & 0.807 \\
11 & 681 & 857 & 857 & 1076 & 0.712 \\
12 & 912 & 1064 & 1092 & 1092 & 0.812 \\
13 & 1289 & 1601 & 1601 & 1709 & 0.743 \\
14 & 1666 & 1958 & {$[1958]$} & 3075 & 0.818 \\
15 & 2241 & 2834 & {$[2834]$} & & 0.744 \\
16 & 2816 & 3312 & {$[3376]$} & 3522 & $\underline{0.824}$ \\
17 & 3649 & 4628 & {$[4633]$} & & 0.752 \\
\cline { 3 - 4 } 18 & 4482 & 5354 & {$[5354]$} & 6242 & 0.817 \\
19 & 5641 & 7081 & {$[7081]$} & & 0.767 \\
20 & 6800 & 8148 & {$[8148]$} & 8840 & 0.818 \\
21 & 8361 & 10552 & {$[10552]$} & & 0.768 \\
22 & 9922 & 11886 & {$[11886]$} & 14102 & 0.821 \\
23 & 11969 & 15154 & {$[15154]$} & & 0.769 \\
24 & 14016 & 16812 & {$[17208]$} & & 0.822 \\
\hline
\end{tabular}

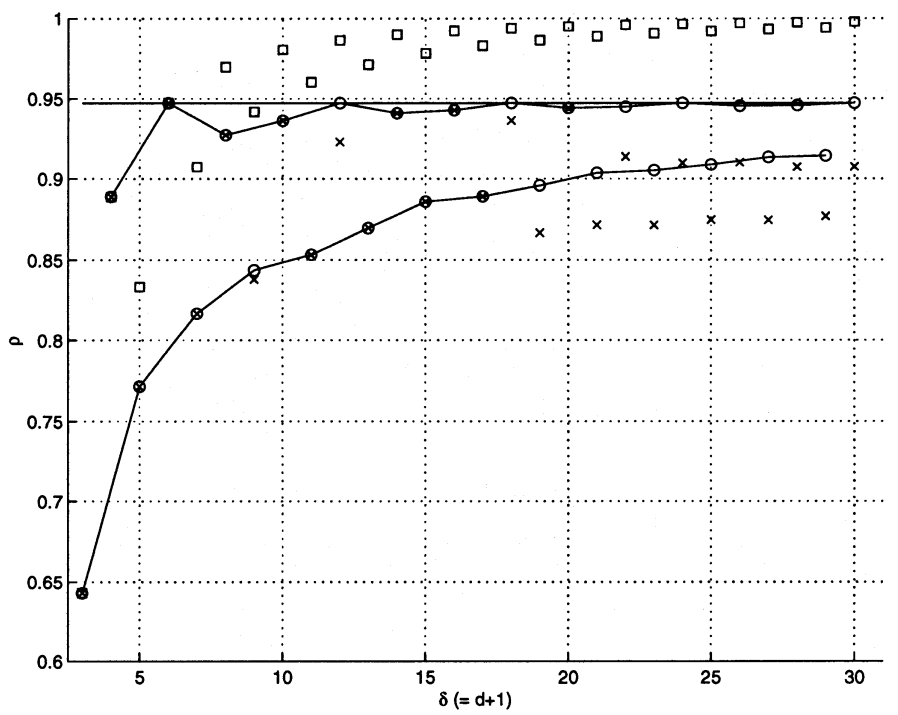

Figure 1. $\rho$ as a function of $\delta$ for three-dimensional rules $(\times$ refers to $N_{\text {prev }}, \circ$ refers to $N_{K O}, \square$ refers to $N_{M E}$, the line at $\rho=18 / 19$ refers to $N_{C L}$ ) 


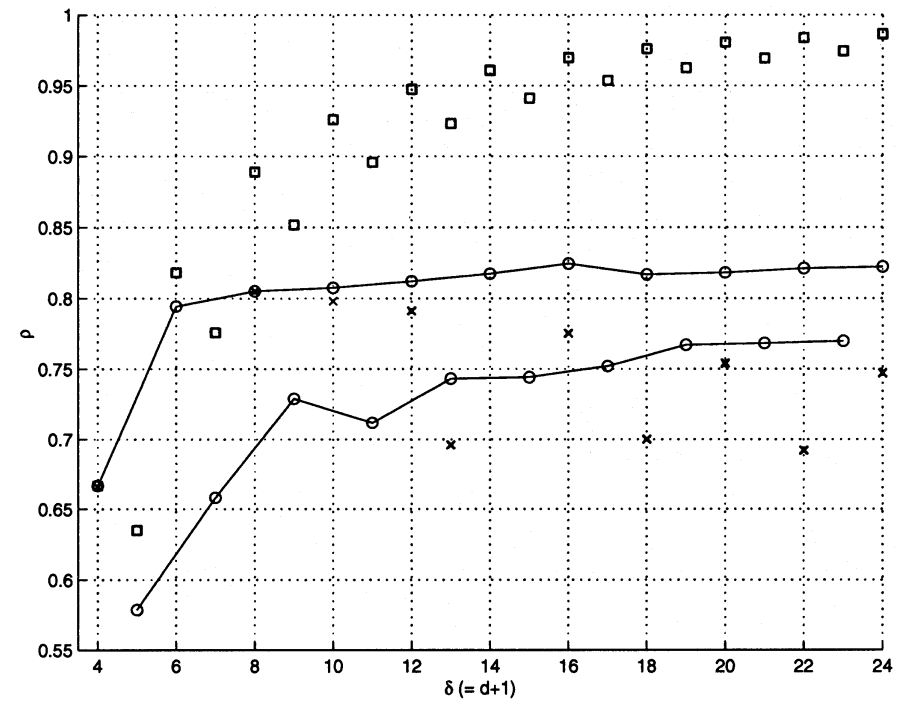

Figure 2. $\rho$ as a function of $\delta$ for four-dimensional rules $(\times$ refers to $N_{\text {prev }}$, o refers to $N_{K O}, \square$ refers to $N_{M E}$ )

In Figures 1 and 2 we present much of the material in Tables 1 and 2 graphically. For any abscissa count $N$, we can calculate the associated packing factor

$$
\rho(N):=\frac{\delta^{s}}{s ! N} .
$$

This is a measure of the efficiency of any rule $Q(\Lambda)$ of enhanced degree $\delta$ and abscissa count $N$ and is the packing factor of the dual lattice $\Lambda^{\perp}$. The packing factor is bounded by $\theta(s)$. In the final section we shall illustrate our discussion of some of these results using these figures.

Many of the entries in the tables specify rank- 1 simple rules. When $Q(\Lambda)$ is an $s$-dimensional rank-1 simple rule, the Hermite normal form (see (2.1) above) of the generator matrix of $\Lambda^{\perp}$ has a readily recognisable form as its principal minor coincides with the identity matrix. The $D-Z$ form (see (1.1)) of this rule is then $Q[1, D, \mathbf{z}, s]$ with

$$
D=N=H_{s, s} ; \quad \mathbf{z}=\left(N-H_{1, s}, N-H_{2, s}, \ldots, N-H_{(s-1), s}, 1\right) .
$$

Naturally, this is in the same equivalence class as the rule specified by

$$
D=N=H_{s, s} ; \quad \mathbf{z}=\left(1, H_{1, s}, H_{2, s}, \ldots, H_{(s-1), s}\right) .
$$

4.2. Three-dimensional lattice rules. For every abscissa count we have listed, we have specified at least one cubature rule. Table 3 contains specifications of thirtyone $K$-optimal rules. This list is complete in the sense that every $K$-optimal rule of enhanced degree thirty or less is included here or is symmetrically equivalent to one listed here. This specification comprises the nontrivial elements of the Hermite normal form of $\Lambda^{\perp}$ (unique to the rule). See (2.1) above. 
THREE- AND FOUR-DIMENSIONAL $K$-OPTIMAL LATTICE RULES

TABLE 3. Three-dimensional $K$-optimal lattice rules

\begin{tabular}{|r|r|rrrrrr|l|l|}
\hline & & Hermite Normal Form of Dual Lattice & & \\
$\delta$ & $N$ & $H_{11}$ & $H_{12}$ & $H_{13}$ & $H_{22}$ & $H_{23}$ & $H_{33}$ & $\mu$ & Rank \\
\hline 2 & 2 & 1 & 0 & 1 & 1 & 1 & 2 & 1 & 1 \\
3 & 7 & 1 & 0 & 2 & 1 & 3 & 7 & 8 & 1 \\
4 & 12 & 1 & 0 & 3 & 1 & 5 & 12 & 12 & 1 \\
5 & 27 & 1 & 0 & 4 & 1 & 10 & 27 & 24 & 1 \\
& & 1 & 1 & 4 & 3 & 6 & 9 & 4 & 2 \\
6 & 38 & 1 & 0 & 7 & 1 & 11 & 38 & 8 & 1 \\
7 & 70 & 1 & 0 & 16 & 1 & 25 & 70 & 24 & 1 \\
8 & 92 & 1 & 0 & 9 & 1 & 39 & 92 & 24 & 1 \\
9 & 144 & 1 & 1 & 11 & 4 & 16 & 36 & 8 & 2 \\
10 & 178 & 1 & 0 & 11 & 1 & 75 & 178 & 24 & 1 \\
11 & 260 & 1 & 0 & 40 & 1 & 94 & 260 & 24 & 1 \\
& & 1 & 0 & 48 & 2 & 56 & 130 & 24 & 2 \\
12 & 304 & 2 & 0 & 14 & 2 & 22 & 76 & 8 & 3 \\
13 & 421 & 1 & 0 & 16 & 1 & 182 & 421 & 24 & 1 \\
14 & 486 & 1 & 0 & 41 & 1 & 57 & 486 & 24 & 1 \\
15 & 635 & 1 & 0 & 146 & 1 & 274 & 635 & 8 & 1 \\
16 & 724 & 1 & 0 & 49 & 1 & 79 & 724 & 24 & 1 \\
17 & 921 & 1 & 0 & 81 & 1 & 222 & 921 & 24 & 1 \\
18 & 1026 & 3 & 0 & 21 & 3 & 33 & 114 & 8 & 3 \\
19 & 1276 & 1 & 0 & 222 & 1 & 538 & 1276 & 24 & 1 \\
20 & 1412 & 1 & 0 & 59 & 1 & 665 & 1412 & 24 & 1 \\
21 & 1708 & 1 & 1 & 121 & 2 & 338 & 854 & 8 & 2 \\
22 & 1878 & 1 & 0 & 75 & 1 & 731 & 1878 & 24 & 1 \\
23 & 2240 & 1 & 0 & 166 & 4 & 255 & 560 & 24 & 1 \\
24 & 2432 & 4 & 0 & 28 & 4 & 44 & 152 & 8 & 3 \\
25 & 2865 & 1 & 0 & 222 & 1 & 965 & 2865 & 24 & 1 \\
26 & 3098 & 1 & 0 & 423 & 1 & 1299 & 3098 & 24 & 1 \\
27 & 3591 & 1 & 0 & 278 & 1 & 1718 & 3591 & 8 & 1 \\
28 & 3868 & 1 & 0 & 205 & 1 & 975 & 3868 & 24 & 1 \\
29 & 4445 & 1 & 0 & 750 & 1 & 1635 & 4445 & 24 & 1 \\
30 & 4750 & 5 & 0 & 35 & 5 & 55 & 190 & 8 & 3 \\
\hline
\end{tabular}

The penultimate column contains $\mu$, the number of distinct rules (symmetric copies) in the symmetry group that contains the listed rule. These may be obtained from the listed rule by coordinate reversal and interchange. Naturally, we list only one rule of the $\mu$ possibilities. This is chosen to be the first in a lexicographic ordering based on the diagonal elements, followed by the nondiagonal elements in the order used in the table. In the language of [LS93, this provides a senior. Also, if the rank is 1, this provides a rank-1 simple rule, unless there happens to be no rank-1 simple rule in the set.

The eight rules of enhanced degree $\delta=6 k$ with $k>1$ are simply $k$-copy versions of the eight rules of enhanced degree 6 . These are of rank 3 .

A supplementary list of three-dimensional optimal rank-1 simple lattice rules is given in Table 4 This list is of the same character as the previous list. It includes all optimal rank-1 simple rules for those degrees for which such a rule does not appear in the previous list. 
TABLE 4. Three-dimensional optimal rank-1 simple lattice rules

\begin{tabular}{|c|c|c|c|c|c|c|c|c|}
\hline \multirow[b]{2}{*}{$\delta$} & \multirow[b]{2}{*}{$N$} & \multicolumn{6}{|c|}{ Hermite Normal Form of Dual Lattice } & \multirow[b]{2}{*}{$\mu$} \\
\hline & & $H_{11}$ & $H_{12}$ & $H_{13}$ & $H_{22}$ & $H_{23}$ & $H_{33}$ & \\
\hline 9 & 145 & 1 & 0 & 9 & 1 & 61 & 145 & 24 \\
\hline \multirow[t]{2}{*}{12} & 312 & 1 & 0 & 13 & 1 & 115 & 312 & 24 \\
\hline & & 1 & 0 & 29 & 1 & 67 & 312 & 24 \\
\hline \multirow[t]{2}{*}{18} & 1038 & 1 & 0 & 35 & 1 & 365 & 1038 & 24 \\
\hline & & 1 & 0 & 119 & 1 & 421 & 1038 & 24 \\
\hline \multirow[t]{2}{*}{21} & 1723 & 1 & 0 & 24 & 1 & 464 & 1723 & 24 \\
\hline & & 1 & 0 & 79 & 1 & 755 & 1723 & 24 \\
\hline 23 & 2255 & 1 & 0 & 100 & 1 & 172 & 2255 & 24 \\
\hline \multirow[t]{2}{*}{24} & 2448 & 1 & 0 & 185 & 1 & 1081 & 2448 & 24 \\
\hline & & 1 & 0 & 199 & 1 & 479 & 2448 & 24 \\
\hline \multirow[t]{2}{*}{30} & 4770 & 1 & 0 & 131 & 1 & 689 & 4770 & 24 \\
\hline & & 1 & 0 & 101 & 1 & 1339 & 4770 & 24 \\
\hline
\end{tabular}

4.3. Four-dimensional lattice rules. We have reported our four-dimensional results in almost the same way as the three-dimensional results. The differences arise from having to curtail our effort because of the higher computational expense. As in the three-dimensional case, we have specified in Table 5 the optimal rules we have found. As mentioned in Section 3, any of these may be actual optimal rules of the stated enhanced degree. We have found all the $K$-optimal rules for $\delta \in[1,13]$, all the $K\left(4, \delta ; q_{1}\right)$-optimal rules for $\delta \in[14,17]$, and all the $K\left(4, \delta ; q_{1}^{+}\right)$-optimal rules

TABLE 5. Four-dimensional $K$-optimal lattice rules

\begin{tabular}{|c|c|c|c|c|c|c|c|c|c|c|c|c|c|}
\hline \multirow[b]{2}{*}{$\delta$} & \multicolumn{11}{|c|}{ Hermite Normal Form of Dual Lattice } & \multirow[b]{2}{*}{$\mu$} & \multirow[b]{2}{*}{ Rank } \\
\hline & $N$ & $H_{11}$ & $H_{12}$ & $H_{13}$ & $H_{14}$ & $H_{22}$ & $H_{23}$ & $\mathrm{H}_{24}$ & $H_{33}$ & $H_{34}$ & $H_{44}$ & & \\
\hline \multicolumn{14}{|c|}{$\overline{\text { Full Search over } K(4, \delta)}$} \\
\hline 1 & 1 & 1 & 0 & 0 & 0 & 1 & 0 & 0 & 1 & 0 & 1 & 1 & 1 \\
\hline 2 & 2 & 1 & 0 & 0 & 1 & 1 & 0 & 1 & 1 & 1 & 2 & 1 & 1 \\
\hline \multirow[t]{2}{*}{3} & 9 & 1 & 0 & 1 & 1 & 1 & 1 & 2 & 3 & 0 & 3 & 8 & 2 \\
\hline & & 1 & 0 & 0 & 2 & 1 & 0 & 3 & 1 & 4 & 9 & 64 & 1 \\
\hline \multirow[t]{4}{*}{4} & 16 & 1 & 1 & 1 & 1 & 2 & 0 & 2 & 2 & 2 & 4 & 2 & 3 \\
\hline & & 1 & 0 & 1 & 2 & 1 & 2 & 1 & 4 & 0 & 4 & 12 & 2 \\
\hline & & 1 & 0 & 0 & 3 & 1 & 1 & 2 & 2 & 6 & 8 & 24 & 2 \\
\hline & & 1 & 0 & 0 & 3 & 1 & 0 & 5 & 1 & 7 & 16 & 48 & 1 \\
\hline 5 & 45 & 1 & 0 & 0 & 4 & 1 & 1 & 6 & 3 & 9 & 15 & 24 & 2 \\
\hline 6 & 68 & 1 & 0 & 0 & 13 & 1 & 1 & 6 & 2 & 16 & 34 & 48 & 2 \\
\hline 7 & 152 & 1 & 0 & 0 & 16 & 1 & 0 & 28 & 1 & 37 & 152 & 96 & 1 \\
\hline 8 & 212 & 1 & 0 & 0 & 9 & 1 & 0 & 33 & 1 & 87 & 212 & 192 & 1 \\
\hline 9 & 375 & 1 & 1 & 1 & 6 & 5 & 0 & 10 & 5 & 10 & 15 & 24 & 3 \\
\hline 10 & 516 & 1 & 0 & 0 & 15 & 1 & 0 & 83 & 2 & 118 & 258 & 192 & 2 \\
\hline 11 & 857 & 1 & 0 & 0 & 188 & 1 & 0 & 207 & 1 & 351 & 857 & 48 & 1 \\
\hline 12 & 1064 & 1 & 0 & 0 & 153 & 1 & 0 & 259 & 2 & 98 & 532 & 96 & 2 \\
\hline 13 & 1601 & 1 & 0 & 0 & 40 & 1 & 0 & 310 & 1 & 408 & 1601 & 48 & 1 \\
\hline \multicolumn{14}{|c|}{ Full Search over $K\left(4, \delta ; q_{1}\right)$} \\
\hline 14 & 1958 & 1 & $\overline{0}$ & 0 & 107 & 1 & 0 & 229 & 1 & 525 & 1958 & 192 & 1 \\
\hline \multirow[t]{2}{*}{15} & 2834 & 1 & 0 & 0 & 892 & 1 & 0 & 1123 & 1 & 1314 & 2834 & 96 & 1 \\
\hline & & 1 & 0 & 0 & 294 & 1 & 1 & 117 & 2 & 507 & 1417 & 96 & 1. \\
\hline 16 & 3312 & 1 & 0 & 0 & 495 & 1 & 0 & 737 & 2 & 450 & 1656 & 96 & $\begin{array}{l}\text { (not simple) } \\
2\end{array}$ \\
\hline 17 & 4628 & 1 & 0 & 0 & 1123 & 1 & 1 & 327 & 2 & 1032 & 2314 & 96 & 2 \\
\hline \multicolumn{14}{|c|}{ Full Search over $K\left(4, \delta ; q_{1}^{+}\right)$} \\
\hline 18 & 5354 & 1 & 0 & 0 & 83 & 1 & 0 & 1253 & 1 & 1863 & 5354 & 192 & 1 \\
\hline 19 & 7081 & 1 & 0 & 0 & 241 & 1 & 0 & 1433 & 1 & 1616 & 7081 & 48 & 1 \\
\hline 20 & 8148 & 1 & 0 & 0 & 371 & 1 & 0 & 1401 & 1 & 3299 & 8148 & 192 & 1 \\
\hline 21 & 10552 & 1 & 0 & 0 & 1670 & 1 & 0 & 2111 & 1 & 2746 & 10552 & 192 & 1 \\
\hline 22 & 11886 & 1 & 0 & 0 & 457 & 1 & 0 & 3753 & 1 & 4079 & 11886 & 192 & 1 \\
\hline 23 & 15154 & 1 & 0 & 0 & 2602 & 1 & 0 & 6037 & 1 & 6424 & 15154 & 96 & 1 \\
\hline 24 & 16812 & 1 & 0 & 0 & 109 & 1 & 1 & 1717 & 3 & 1677 & 5604 & 192 & 2 \\
\hline
\end{tabular}


TABLE 6. Four-dimensional rank-1 simple lattice rules

\begin{tabular}{|r|r|rrrrrrrrrr|r|r|}
\hline & & \multicolumn{10}{|c|}{ Hermite Normal Form of Dual Lattice } & \\
$\delta$ & $N$ & $H_{11}$ & $H_{12}$ & $H_{13}$ & $H_{14}$ & $H_{22}$ & $H_{23}$ & $H_{24}$ & $H_{33}$ & $H_{34}$ & $H_{44}$ & $\mu$ \\
\hline 9 & 398 & 1 & 0 & 0 & 8 & 1 & 0 & 61 & 1 & 149 & 398 & 192 \\
16 & 3376 & 1 & 0 & 0 & 169 & 1 & 0 & 1091 & 1 & 1387 & 3376 & 192 \\
17 & 4633 & 1 & 0 & 0 & 547 & 1 & 0 & 1936 & 1 & 1965 & 4633 & 48 \\
24 & 17208 & 1 & 0 & 0 & 919 & 1 & 0 & 4701 & 1 & 5557 & 17208 & 192 \\
\hline
\end{tabular}

for $\delta \in[18,24]$. The supplementary Table 6 simply specifies some rank-1 simple rules whose abscissa counts appear in Table 2 but are not specified elsewhere.

\section{Further COMments}

Any historical perspective on rules of specified trigonometrical degree would mention the widespread use of the product trapezoidal rule, and the center and vertex rule since the beginning of the twentieth century. However, the serious study of such rules seems to have started in the final fifteen years of that century. The earlier work of this period, mainly by Russian authors, has been strictly limited to rank-1 simple rules. They have produced and established the optimal degree rules up to $\delta=4$. These authors have been concerned mainly with rule families in three, four, and five dimensions. Each family contains rules of arbitrarily high degree. Other economical rules seem to have been provided only as spin-off, and no claim has been made for optimality. However, in retrospect we have ascertained that in three dimensions their rules are optimal rank- 1 simple rules for all $\delta \leq 18$ but that in four dimensions, they are optimal only for odd $\delta$ up to 5 and for even $\delta$ up to 12 .

To our knowledge, the only other set of rules proposed in this context are the Smolyak rules CNR99. These were designed for high dimensions and high degrees. In three and four dimensions and for values of $\delta$ considered here, the $K$-optimal rules presented here are well over ten times more cost effective than the corresponding Smolyak rules.

Figures 11 and 2 illustrate most of the abscissa counts listed in Tables 11 and 2 We note the dichotomy between even and odd degree, which seems to occur in both the theoretical limit $N_{M E}$ and results such as $N_{K O}$ and $N_{r 1 s}$ (not shown in figures but reported in the tables) and $N_{\text {prev }}$.

As discussed in Section 1, we have no theory to exclude the possibility that, for larger $\delta$, the optimal rule of trigonometric degree $\delta$ is not a lattice rule. If this were the case, in Figures 1 and 2 there would be missing entries above the lines joining the circles, but below the theoretical limit represented by squares. Also unsatisfactory is the fact that we cannot establish that the $K$-optimal lattice rule is actually an optimal lattice rule. This is more frustrating because the anecdotal evidence is overwhelming. We have several incomplete proofs, characterized by our inability to bridge in each case what seems to be a minor lacuna. However, we have an example of a rule that is $K(4, \delta)$-optimal, but not $K\left(4, \delta ; q_{2}\right)$-optimal. The 375 point lattice listed in Table 5 for $\delta=9$ is not in $K\left(4,9 ; q_{2}\right)$. The $K\left(4,9 ; q_{2}\right)$-optimal rules have an abscissa count of 390 . And we have encountered many examples in which the restriction to $K\left(4, \delta ; q_{1}^{+}\right)$has resulted in missing some excellent rules. 
One of the unsatisfactory features of our approach is its high computational cost. We have derived a somewhat unrealistic upper bound on the complexity. This depends in the first place on $\nu$, the number of distinct generator matrices we start with. As specified in Section 3 .

$$
\nu=\left(\begin{array}{c}
s+\delta-1 \\
s-1
\end{array}\right)^{s}=\mathcal{O}\left(\delta^{s^{2}-s}\right)
$$

for fixed $s$ and increasing $\delta$.

Only a proportion that appears to decrease with increasing $\delta$ is treated further to find $N$. After this, a minute proportion of these are retained to find their degree. A simple basic form of our algorithm to determine the degree of a lattice rule requires time proportional to $\bar{\delta}^{s-1}$, where $\bar{\delta}$ is the degree of the lattice. In fact, all but a handful have degree strictly less than $\delta$. To obtain a complexity bound, we replace both proportions by 1 and replace $\bar{\delta}$ by $\delta$. This approach leads to a complexity bounded above by $\delta^{s^{2}-1}$.

For the values of $\delta$ for which we carried out careful timing checks, the computational cost does increase very rapidly with increasing $\delta$, although not nearly so rapidly as the complexity bound derived above might suggest. To give the reader an idea, we list some timings below for a particular processor 1

- For $s=3$ all $\delta \leq 30$ are treated within 33 minutes.

- For $s=4$ all $\delta \leq 8$ are treated within 34 minutes.

- For $s=4$ and $\delta=10$ the search required 6.5 hours.

- For $s=4$ and $\delta=14$ the search restricted to $K\left(4,14 ; q_{1}\right)$ required 120 hours.

- For $s=4$ and $\delta=17$ the search restricted to $K\left(4,17 ; q_{1}^{+}\right)$required 145 hours.

- For $s=4$ and $\delta=18$ the search restricted to $K\left(4,18 ; q_{1}^{+}\right)$required 228 hours.

In fact, higher values of $\delta$ were treated in a different way by partitioning the search into several tasks that were distributed to several different machines. Using actual timings, we estimated hypothetical timings corresponding to the chip mentioned above. These indicated that the time needed for a complete search for $\delta=20$ would be about 2700 days, but restricting the search to $K\left(4,20 ; q_{1}^{+}\right)$reduced this time to about 40 days.

Another feature of our program is its exorbitant redundancy. In an extreme case, a four-dimensional lattice may have 30 points on $\Omega(4, \delta)$, these comprising two point pairs on each of seven facet pairs, and one point pair on the remaining facet pair. When $q_{1}$ includes four of these facet pairs, our search over $K\left(4, \delta, q_{1}\right)$ may include the identical lattice sixteen times. Moreover, we might treat each of the 192 lattices in the same equivalence class either eight or sixteen times. All this work might provide a single entry in Table 5. This helps us to understand why the much smaller population space $K\left(4, \delta, q_{1}^{+}\right)$often but not always includes at least one of the set of $K$-optimal lattices associated with the larger (by a factor of up to 24) set. We note that the complexity or the complexity bound would not be affected by this redundancy. It shows itself in the circumstance that an optimal rule was usually found in the first hour of a 100-hour run.

\footnotetext{
${ }^{1}$ Pentium II (Deschutes), 398.13 bogomips processor
} 
For some parts of the search, this redundancy is not important. As an analogy one might compare the task of searching for one of $k$ needles in one haystack with that of searching for one of $100 k$ needles in 100 mixed-up haystacks. So long as $k \geq 1$, the time taken to find one needle is to first order the same in either case. If $k=0$, it takes 100 times as long to complete the search in the second case as in the first case.

\section{Appendix: Specification of Sets Defined in Theorem 3.4}

The group $\mathcal{G}$ of coordinate transformations includes transpositions $\sigma_{i j}$ that interchange coordinates $x_{i}$ and $x_{j}$ and reflections $\rho_{i}$ that replace $x_{i}$ by $-x_{i}$. The group can be generated by the four elements $\sigma_{12}, \sigma_{13}, \sigma_{14}$ and $\rho_{3}$, which we have temporarily termed $\mathcal{G}_{i}(i=1,2,3,4)$. Hence, we can establish the theorem by exploiting the result that $\mathcal{G}_{i} q \in S_{j}(i=1,2,3,4)$ whenever $q \in S_{j}$.

The effect of each of these four transformations on each of the eight facet-pairs is given in the following table.

\begin{tabular}{|c|c|c|c|c|c|c|c|}
\hline & Specification & Parity & & $\sigma_{12}$ & & $\sigma_{14}$ & $\rho_{3}$ \\
\hline$F_{0}$ & $\begin{array}{llll}+, & +, & +, & +\end{array}$ & $\mathrm{E}$ & $F_{0}$ & $F_{0}$ & $F_{0}$ & $F_{0}$ & $F_{4}$ \\
\hline$F_{1}$ & $\begin{array}{llll}-, & +, & +\end{array}$ & $\mathrm{O}$ & $F_{1}$ & $F_{2}$ & $F_{4}$ & $F_{7}$ & $F_{5}$ \\
\hline$F_{2}$ &,$+ \quad-, \quad+$, & $\mathrm{O}$ & $F_{2}$ & $F_{1}$ & $F_{2}$ & $F_{2}$ & $F_{6}$ \\
\hline$F_{3}$ &,$- \quad-, \quad+$, & $\mathrm{E}$ & $F_{3}$ & $F_{3}$ & $F_{6}$ & $F_{5}$ & $F_{7}$ \\
\hline$F_{4}$ &,$+ \quad+, \quad-$, & $\mathrm{O}$ & $F_{4}$ & $F_{4}$ & $F_{1}$ & $F_{4}$ & $F_{0}$ \\
\hline$F_{5}$ &,$- \quad+, \quad-$, & $\mathrm{E}$ & $F_{5}$ & $F_{6}$ & $F_{5}$ & $F_{3}$ & $F_{1}$ \\
\hline$F_{6}$ &,$+ \quad-$, & $\mathrm{E}$ & $F_{6}$ & $F_{5}$ & $F_{3}$ & $F_{6}$ & $F_{2}$ \\
\hline$F_{7}$ &,$- \quad-$, & $\mathrm{O}$ & $F_{7}$ & $F_{7}$ & $F_{7}$ & $F_{1}$ & $F_{3}$ \\
\hline
\end{tabular}

To illustrate the calculation, we confirm the entry for $\sigma_{13} F_{3}$. By definition, $F_{3}$ includes only points of the form $(-a,-b, c, d)$ where $a, b, c$, and $d$ are individually nonnegative. The corresponding point of $\sigma_{13} F_{3}$ is obtained by interchange of coordinates 1 and 3 , and so is $(c,-b,-a, d)$. Reference to the definition confirms that this point is indeed an element of $F_{6}$. Thirty-two equally trivial calculations will confirm the results presented in this table.

Using these operations, we can transform quartets of facet-pairs into other quartets. We overload the notation above and consider the order of facet-pairs in a quartet as irrelevant; for example, $q(7,1,4,2)=q(1,2,4,7)$.

One way to obtain one of the sets listed below is by constructing a list as follows.

Initially this list contains only one element, in this case perhaps $q_{2 a}=q(0,2,4,6)$. At the end of a later stage, it may contain $N$ distinct elements, say, $q_{1}, q_{2}, \ldots, q_{N}$. The next stage comprises calculating $G_{i} q_{k}$ for $i=1,2,3,4$ and $k=1,2, \ldots, N$, adding these to the list and removing duplicates. If the new list has more than $N$ elements, we proceed to a further stage of the same nature. If the new list has $N$ elements, the same number as in the previous list, we may stop. The current list now comprises a complete list of the elements of $S_{2 a}$.

Again, we illustrate one of these calculations by an example. We evaluate $\sigma_{13} q(0,2,4,6)$. We require from the table the facet-pairs $\sigma_{13} F_{j}$ for $j=0,2,4,6$. Reference to the column headed $\sigma_{13}$ of the table shows these to be $F_{0}, F_{2}, F_{1}, F_{3}$, respectively. These facet-pairs comprise $q(0,2,1,3)$, which is the same as $q(0,1,2,3)$. 
Each update of the list involves four such calculations for each of the current $N$ members of the list.

- Elements of $S_{0}$

$$
q(0,3,5,6), \quad q(1,2,4,7)
$$

- Elements of $S_{1}$

$$
\begin{array}{llll}
q(0,2,4,7), & q(0,1,4,7), & q(0,1,2,7), & q(0,1,2,4), \\
q(0,3,4,6), & q(0,4,5,6), & q(0,1,3,5), & q(0,2,3,6), \\
q(0,3,4,5), & q(0,1,3,6), & q(0,5,6,7), & q(0,3,5,7), \\
q(0,3,6,7), & q(0,1,5,6), & q(0,2,5,6), & q(0,2,3,5), \\
q(3,4,5,6), & q(1,4,5,7), & q(2,4,6,7), & q(1,2,3,4), \\
q(1,2,4,6), & q(1,2,4,5), & q(1,2,3,7), & q(2,4,5,7), \\
q(1,4,6,7), & q(1,2,5,7), & q(1,2,6,7), & q(2,3,4,7), \\
q(1,3,4,7), & q(2,3,5,6), & q(1,3,5,6), & q(3,5,6,7) \\
q \text { ments of } S_{2 a} & & \\
q(0,2,4,6), & q(0,1,4,5), & q(0,1,2,3), & q(0,3,4,7), \\
q(0,1,6,7), & q(0,2,5,7), & q(2,3,4,5), & q(1,3,4,6), \\
q(1,2,5,6), & q(2,3,6,7), & q(1,3,5,7), & q(4,5,6,7)
\end{array}
$$

- Elements of $S_{2 a}$

- Elements of $S_{2 b}$

$$
\begin{array}{llll}
q(0,2,4,5), & q(0,1,4,6), & q(0,1,2,5), & q(0,2,3,4), \\
q(0,1,3,4), & q(0,1,2,6), & q(0,4,6,7), & q(0,4,5,7), \\
q(0,1,3,7), & q(0,2,3,7), & q(0,2,6,7), & q(0,1,5,7), \\
q(2,4,5,6), & q(1,4,5,6), & q(1,2,3,6), & q(1,2,3,5), \\
q(2,3,4,6), & q(1,3,4,5), & q(2,5,6,7), & q(1,5,6,7), \\
q(2,3,5,7), & q(1,3,6,7), & q(3,4,6,7), & q(3,4,5,7)
\end{array}
$$

\section{ACKNOWLEDGMENT}

It is a pleasure to acknowledge the significant interest and help of Dr. T. Canfield in setting up some of these highly parallel programs.

\section{REFERENCES}

[BC93] M. Beckers and R. Cools, A relation between cubature formulae of trigonometric degree and lattice rules, International Series of Numerical Mathematics., Vol. 112, Numerical Integration IV (H. Brass and G. Hämmerlin, eds.), Birkhäuser Verlag, Basel, 1993, pp. 13-24. MR 95b:65034

[CNR99] R. Cools, E. Novak, and K. Ritter, Smolyak's construction of cubature formulas of arbitrary trigonometric degree, Computing 62, no. 2, (1999), 147-162. MR 2000c:41041

[CR97] R. Cools and A. Reztsov, Different quality indexes for lattice rules, J. Complexity 13 (1997), 235-258. MR 98e:65011

[CS96] R. Cools and I. H. Sloan, Minimal cubature formulae of trigonometric degree, Math. Comp. 65, no. 216, (1996), 1583-1600. MR 97a:65025

[Fro77] K. K. Frolov, On the connection between quadrature formulas and sublattices of the lattice of integral vectors, Dokl. Akad. Nauk SSSR 232 (1977), 40-43, (Russian) Soviet Math. Dokl. 18 (1977), 37-41 (English). MR 55:272

[GL87] P. M. Gruber and C. G. Lekkerkerker, Geometry of numbers, North-Holland, Amsterdam, 1987. MR 88j:11034

[KR95] B. V. Klyuchnikov and A. V. Reztsov, A relation between cubature formulas and densest lattice packings, East J. Approx. 1, no. 4, (1995), 557-570. MR 97g:41045

[LS97] J. N. Lyness and I. H. Sloan, Cubature rules of prescribed merit, SIAM J. Numer. Anal. 34, no. 2, (1997), 586-602. MR 97m:65052

[LS93] J. N. Lyness and T. Sørevik, Lattice rules by component scaling, Math. Comp. 61, no. 204, (1993), 799-820. MR 94a:65011 
[Lyn88] J. N. Lyness, Some comments on quadrature rule construction criteria, International Series of Numerical Mathematics., Vol. 85, Numerical Integration III (G. Hämmerlin and H. Brass, eds.), Birkhäuser Verlag, Basel, 1988, pp. 117-129. MR 91b:65028

[Lyn89] J. N. Lyness, An introduction to lattice rules and their generator matrices, IMA J. Numer. Anal. 9 (1989), 405-419. MR 91b:65029

[Min67] H. Minkowski, Gesammelte Abhandlungen, Reprint (originally published in 2 volumes, Leipzig, 1911), Chelsea Publishing Company, 1967.

[Mys85] I. P. Mysovskikh, Quadrature formulae of the highest trigonometric degree of accuracy, Zh. Vychisl. Mat. i Mat. Fiz. 25 (1985), 1246-1252 (Russian). U.S.S.R. Comput. Maths. Math. Phys. 25 (1985), 180-184 (English). MR 87b:65030

[Mys87] I. P. Mysovskikh, Cubature formulas that are exact for trigonometric polynomials, Dokl. Akad. Nauk SSSR 296 (1987), 28-31 (Russian). Soviet Math. Dokl. 36 (1988), 229-232 (English). MR 89b:41038

[Mys88] I. P. Mysovskikh, Cubature formulas that are exact for trigonometric polynomials, Metody Vyčisl. 15 (1988), 7-19 (Russian). MR 90a:65050

[Nos85] M. V. Noskov, Cubature formulae for the approximate integration of periodic functions, Metody Vyčisl. 14 (1985), 15-23 (Russian). MR 90f:65038

[Nos88a] M. V. Noskov, Cubature formulae for the approximate integration of functions of three variables, Zh. Vychisl. Mat. Mat. Fiz. 28 (1988), 1583-1586 (Russian). U.S.S.R. Comput. Maths. Math. Phys. 28 (1988), 200-202 (English). MR 90j:65042

[Nos88b] M. V. Noskov, Formulas for the approximate integration of periodic functions, Metody Vyčisl. 15 (1988), 19-22 (Russian). CMP 21:03

[Nos91] M. V. Noskov, On the construction of cubature formulae of higher trigonometric degree, Metody Vyčisl. 16 (1991), 16-23 (Russian).

[NS96] M. V. Noskov and A. R. Semenova, Cubature formulae of high trigonometric accuracy for periodic functions of four variables, Comp. Math. Math. Phys. 36, no. 10, (1996), 1325-1330. MR 97h:65029

[Sem96] A. R. Semenova, Computing experiments for construction of cubature formulae of high trigonometric accuracy, Cubature Formulas and Their Applications (Russian) (Ufa) (M. D. Ramazanov, ed.), 1996, pp. 105-115.

[SJ94] I. H. Sloan and S. Joe, Lattice methods for multiple integration, Oxford University Press, 1994. MR 98a:65026

Department of Computer Science, K. U. Leuven, Celestijnenlaan 200A, B-3001 HevERLEe, Belgium

E-mail address: Ronald.Cools@cs.kuleuven.ac.be

Mathematics and Computer Science Division, Argonne National Laboratory, 9700 South Cass Avenue, Argonne, Illinois 60439 and School of Mathematics, University of New South Wales, Sydney 2052 Australia

E-mail address: lyness@mcs.anl.gov 